\title{
LEVANTAMENTO DAS CONDIÇÕES DE FUNCIONAMENTO DOS SERVIÇOS DE RADIOLOGIA DE HOSPITAIS PÚBLICOS E UNIVERSITÁRIOS DO RIO DE JANEIRO*
}

\author{
Marcos Otaviano da Silva ${ }^{1}$, Antonio Carlos Pires Carvalho ${ }^{2}$, Ana Cecília Pedrosa de Azevedo ${ }^{3}$
}

Resumo Foram realizados testes de controle de qualidade, descritos na Portaria 453/98 do Ministério da Saúde, em sete hospitais públicos do Rio de Janeiro, em um total de 29 tubos de raios X, 30 chassis e 20 vestimentas de proteção individual. Os testes avaliaram o desempenho do gerador de raios $X$ e a geometria do feixe. Foram verificados, também, chassis, "écrans" e negatoscópios, assim como as câmaras claras e escuras. Alguns dos resultados encontrados foram: $55 \%$ dos tubos foram reprovados em exatidão e $15 \%$ em reprodutibilidade de $\mathrm{kVp}$; $53 \%$ foram reprovados em exatidão e $31 \%$ em reprodutibilidade de tempo de exposição; $30 \%$ foram reprovados em camada semi-redutora; $18 \%$ foram reprovados no alinhamento do feixe central; $30 \%$ foram reprovados em coincidência de campos; $40 \%$ foram reprovados na linearidade de exposição; $64 \%$ foram rejeitados no teste de ponto focal. Foram verificadas a guarda inadequada e a falta de recomendação para uso dos aventais plumbíferos. Os resultados dos testes sugerem a necessidade da implementação sistemática de controle de qualidade por um profissional qualificado. A relação de custo-benefício reforça a implantação e a manutenção dos procedimentos contidos e executados neste trabalho, pois tais procedimentos garantiriam a diminuição do custo final do serviço e da dose nos pacientes.

Unitermos: Garantia de qualidade; Controle de qualidade; Radiodiagnóstico.

Abstract Evaluation of working conditions of radiology departments of public and university hospitals in Rio de Janeiro, Brazil.

Quality control tests, according to "Portaria 453/98" of the Brazilian Health Ministry, were applied in seven public hospitals of Rio de Janeiro including 29 X-ray tubes, 30 cassettes and 20 individual protection garments. These tests evaluate mainly the X-ray generator and system geometry. Other tests in the accessories were also performed, such as: cassettes, screens, viewing boxes, darkrooms and clear rooms. In the $k V p$ tests, $55 \%$ of the equipments were disapproved in exactitude and $15 \%$ in reproducibility. Time exactitude and reproducibility were not approved in $53 \%$ and $31 \%$ of the equipments, respectively. The percentages of disapprovement were: for the HVL (half-value layer) $30 \%$, for beam alignment $18 \%$, for field coincidence $30 \%$, for exposure linearity $40 \%$ and for focal spot $64 \%$. It has also been observed that frequently the lead aprons were not kept in appropriate hangers and this procedure may cause damage to these accessories. The results of the test suggest the need of implementation of quality assurance tests in these services. The cost benefit analysis of the results reinforces the importance of the implementation of the recommendations contained in this paper.

Key words: Quality assurance; Quality control; Radiodiagnostic.

\section{INTRODUÇÃO}

Este trabalho trata dos parâmetros que implicam qualidade de instalações que rea-

* Trabalho realizado no Hospital Universitário Clementino Fraga Filho (HUCFF) da Universidade Federal do Rio de Janeiro (UFRJ) Rio de Janeiro, RJ.

1. Aluno do Programa de Pós-Graduação em Radiologia da Faculdade de Medicina da UFRJ.

2. Professor Adjunto Doutor do Departamento de Radiologia, Coordenador Adjunto do Programa de Pós-Graduação em Radiologia da Faculdade de Medicina da UFRJ.

3. Física Doutora do Centro de Estudos de Saúde do TrabaIhador e Ecologia Humana da Escola Nacional de Saúde Pública da Fundação Oswaldo Cruz (CESTEH/ENSP/Fiocruz) e da Faculdade de Medicina da UFRJ.

Endereço para correspondência: Prof. Dr. Antonio Carlos Pires Carvalho. Rua Pereira Nunes, 71, cob-01, Tijuca. Rio de Janeiro, RJ, 20511-120. E-mail: acpcrj@hucff.ufrj.br

Recebido para publicação em 14/5/2003. Aceito, após revisão, em 11/7/2003. lizam exames de radiodiagnóstico médico com equipamentos de raios $\mathrm{X}$ convencionais. Para tal, foi verificada a qualidade dos serviços de radiologia (SR) de alguns hospitais universitários e públicos do estado do Rio de Janeiro.

Entre os vários fatores que influem no bom desempenho de um SR, certamente destacam-se os problemas advindos de falhas mecânicas e/ou eletrônicas dos equipamentos, ora pela ausência de manutenção, ora pela falta de calibração dos equipamentos $^{(\mathbf{1})}$. Outras causas incluem o processamento dos filmes, os acessórios usados na realização dos exames, as vestimentas de proteção individuais, dentre outros. Associada a estes problemas, mas não de menor importância, está a falta de treinamento dos técnicos de radiologia ${ }^{(2)}$.

Foram realizados alguns dos testes de controle de qualidade (CQ) descritos na Portaria 453/98 da Secretaria de Vigilância Sanitária do Ministério da Saúde ${ }^{(3)}$. Os testes realizados nos tubos de raios $\mathrm{X}$ se deram de forma sistemática, sendo executados em todos os tubos de cada um dos SR avaliados, enquanto aqueles feitos nos acessórios se deram na forma de amostragem, uma vez que era inviável para o que se propunha este trabalho, uma abordagem de todo o material, por questões como tempo e para não interferir na rotina dos SR.

Assim, este trabalho visa sugerir a implantação sistemática de um programa de 
garantia de qualidade (PGQ), que, além de atender ao dispositivo legal representado pela Portaria 453/98, contribua para a melhoria de outros fatores de igual, ou até de maior importância, pois minimiza gastos e aumenta a confiabilidade dos exames, uma vez que contribui para o aumento na qualidade da imagem e para a redução da perda de filmes e a repetição de exames ${ }^{(2,4,5)}$. Ainda no que diz respeito à implantação de um PGQ,é essencial citar a diminuição da dose de radiação a que são submetidos os pacientes e os profissionais que trabalham diretamente na realização dos exames ${ }^{(2,4-6)}$.

Outro ponto que deve ser levado em consideração quanto à implantação de um PGQ, sugerido neste trabalho, é que ele considera a criação e ampliação de uma filosofia de trabalho baseada na proteção radiológica, uma vez que se torna fator primordial a inclusão e o estabelecimento de protocolos e condutas ${ }^{(\mathbf{7 , 8})}$.

\section{MATERIAIS E MÉTODOS}

Os hospitais que fizeram parte desta pesquisa são: Hospital Universitário Clementino Fraga Filho - Universidade Federal do Rio de Janeiro (HUCFF-UFRJ), Instituto de Puericultura e Pediatria Martagão Gesteira - Universidade Federal do Rio de Janeiro (IPPMG-UFRJ), Hospital Municipal Miguel Couto (HMMC), Hospital Universitário Antônio Pedro - Universidade Federal Fluminense (HUAP-UFF), Hospital Geral de Nova Iguaçu (HGNI), Instituto Fernandes Figueira (IFF) e Hospital da Lagoa (HL). Estes hospitais são identificados neste trabalho como hospitais A, B, C, D, E, F e G. Para evitar comparações e especulações que fujam ao propósito do trabalho, a identificação dos hospitais por nome da instituição e letras não obedece necessariamente a nenhuma ordem lógica.

Cada um dos hospitais foi visitado algumas vezes, dependendo do número de equipamentos, em diferentes dias e horários, inclusive nos finais de semana, sempre dando prioridade à rotina normal do SR. Em cada hospital visitado foi feita entrevista com o chefe do SR, chefe dos técnicos, técnicos, auxiliares e médicos residentes, quando era cabível, para avaliar as condições de proteção radiológica, a rotina de trabalho e eventuais dificuldades.
Os protocolos dos testes de CQ utilizados neste trabalho foram aqueles que mais são usados na literatura nacional ${ }^{(3,9)} \mathrm{e}$ internacional $^{(\mathbf{1 0 , 1 1 )}}$. Os teste de CQ realizados neste trabalho foram: exatidão do indicador de tensão do tubo $(\mathrm{kVp})$, reprodutibilidade de $\mathrm{kVp}$, exatidão do tempo de exposição, reprodutibilidade do tempo de exposição, camada semi-redutora (CSR), alinhamento do eixo central do feixe de raios $\mathrm{X}$ e exatidão do sistema de colimação, reprodutibilidade da taxa de kerma no ar, linearidade da taxa de kerma no ar com $\mathrm{mAs}$, rendimento do tubo ( $\mathrm{mGy} \mathrm{m}^{2} / \mathrm{mA}$ min.), tamanho do ponto focal, integridade dos acessórios e vestimentas de proteção individual, vedação da câmara escura, resolução "écran"-filme, alinhamento de grade, integridade dos "écrans" e chassis, contato "écran"-filme e condições dos negatoscópios.

Os equipamentos utilizados neste trabalho foram os seguintes: medidor de $\mathrm{kVp}$ Unfors - 9001, medidor de tempo Unfors - 8003, câmara de ionização Rad Check, filtros de alumínio, fotômetro digital Victoreen $07-621$, objeto de teste para avaliar o alinhamento de grade Victoreen 07 - 644, objeto de teste para avaliar a coincidência de campos e alinhamento de feixe Victoreen 07 - 661, objeto de teste para avaliar a resolução de "écran"-filme Victoreen $07-527$, objeto de teste para avaliar o contato "écran"-filme Victoreen 07 - 608, lâmpada ultravioleta Spectroline
977C 01-609 e objeto de teste para avaliar o ponto focal RMI - 112B.

\section{RESULTADOS}

Neste trabalho foram verificados 29 tubos de raios X em sete diferentes SR. Um fato que merece destaque nesta avaliação é que, dos tubos de raios $\mathrm{X}$ verificados, apenas um tinha menos de dez anos de vida útil, sendo também o único aparelho que dispunha de manual técnico.

Foram investigados, ainda, 30 chassis e "écrans" e 20 vestimentas de proteção individual, que foram verificados em quatro hospitais. Neste caso foram escolhidos, aleatoriamente, $40 \%$ dos chassis, "écrans" e vestimentas de proteção individual. Os resultados obtidos foram considerados como índice para todos os demais.

Os Gráficos 1 e 2 mostram o porcentual de equipamentos aprovados e reprovados em cada um dos SR, sendo consideradas todas as salas de cada SR.

Os resultados dos testes de tempo de exposição estão apresentados nos Gráficos 3 e 4.

No Gráfico 5 podemos verificar os resultados porcentuais no teste de CSR.

$\mathrm{O}$ teste de alinhamento do feixe central (Gráfico 6) e dois exemplos de imagens geradas neste teste (Figuras 1 e 2) são apresentados. $\mathrm{O}$ primeiro, de um equipamento que está inadequado (Figura 1), e o outro, de um equipamento adequado (Figura 2).

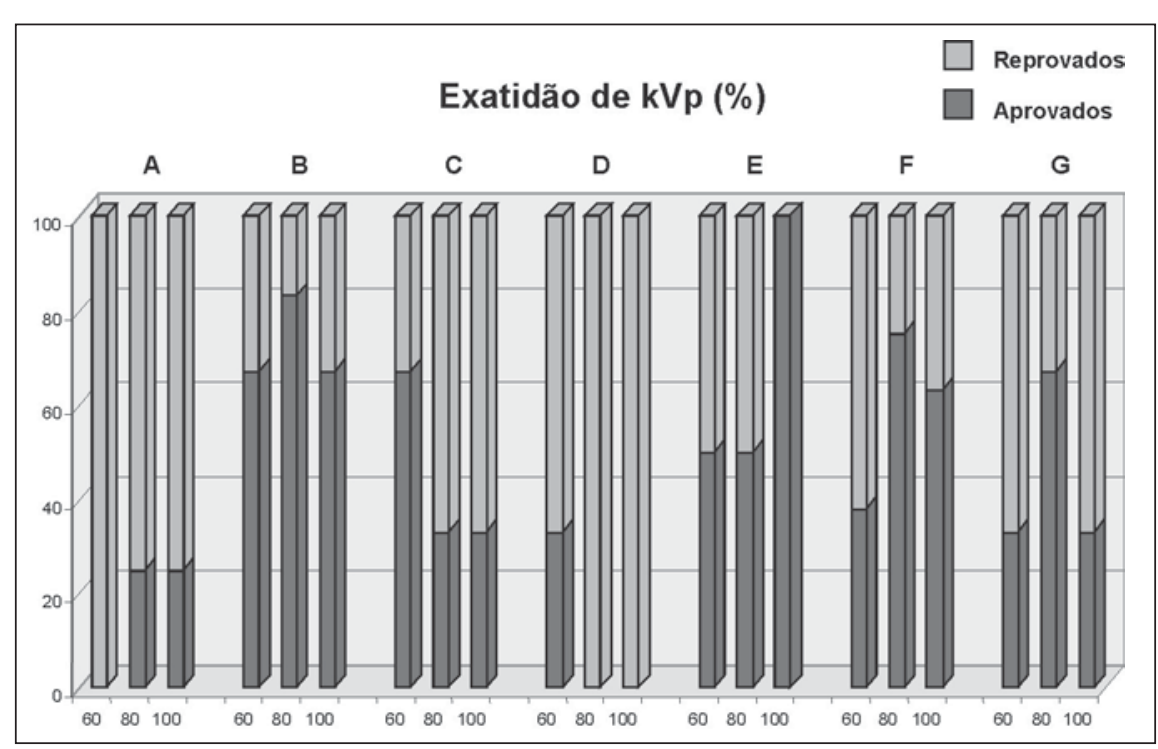

Gráfico 1. Porcentual de equipamentos aprovados e reprovados no teste de exatidão de kVp. 


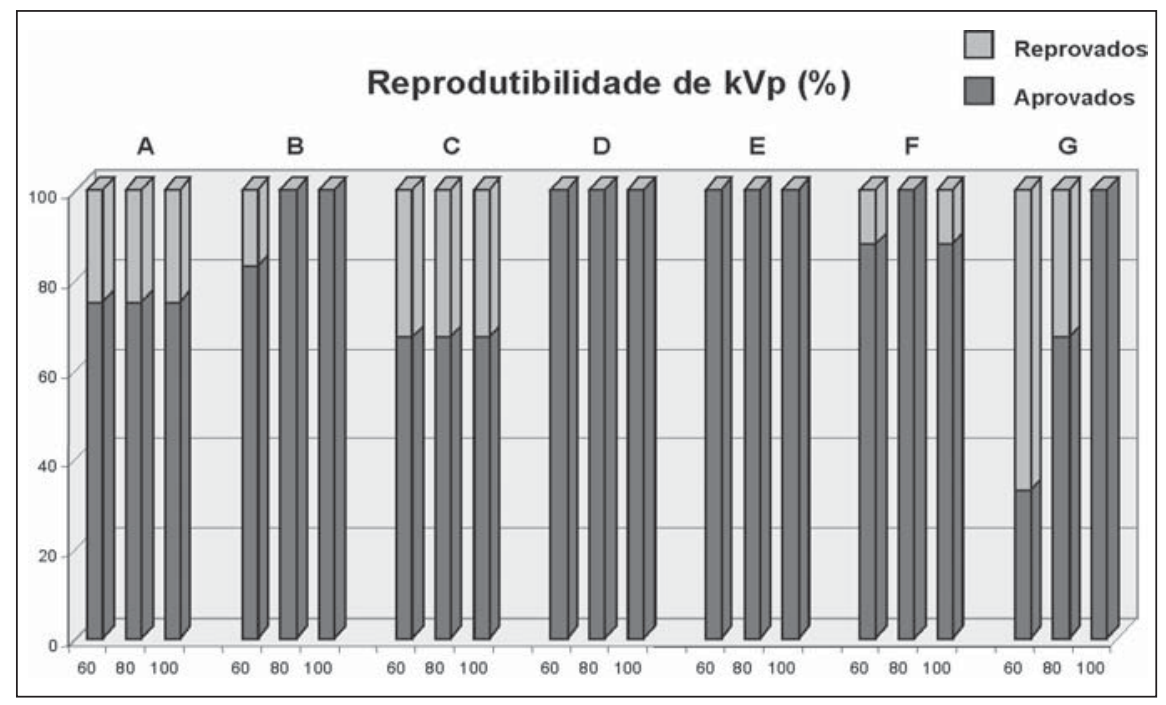

Gráfico 2. Porcentual de equipamentos aprovados e reprovados no teste de reprodutibilidade de kVp.

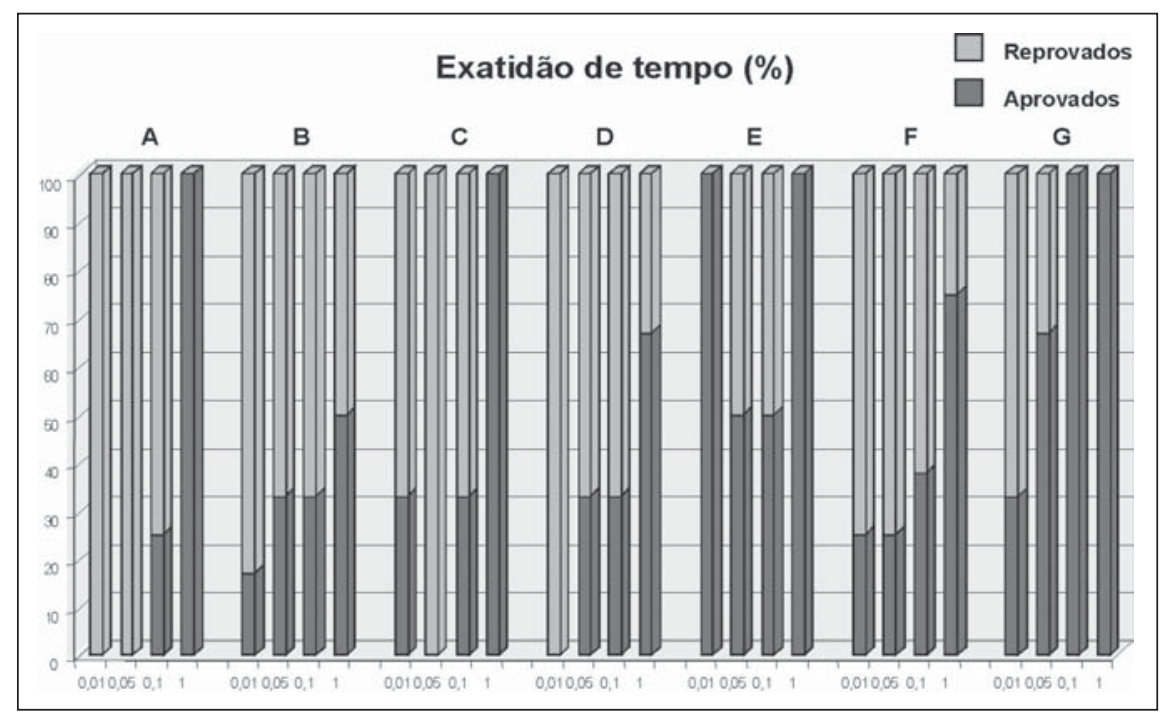

Gráfico 3. Porcentual de equipamentos aprovados e reprovados no teste de exatidão de tempo.

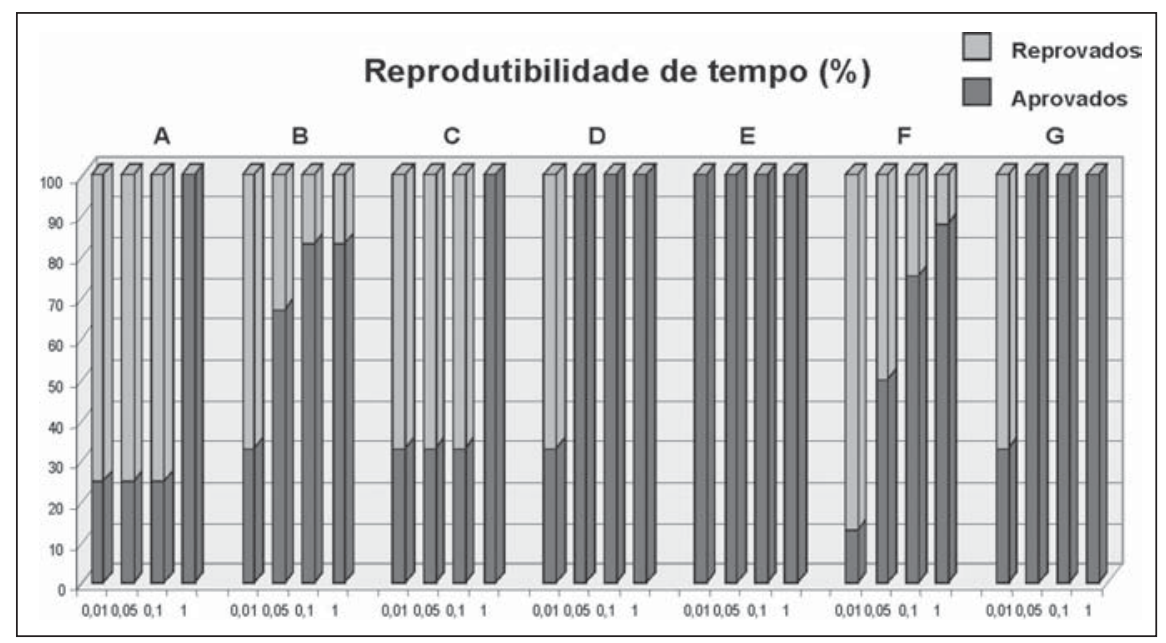

Gráfico 4. Porcentual de equipamentos aprovados e reprovados no teste de reprodutibilidade de tempo.

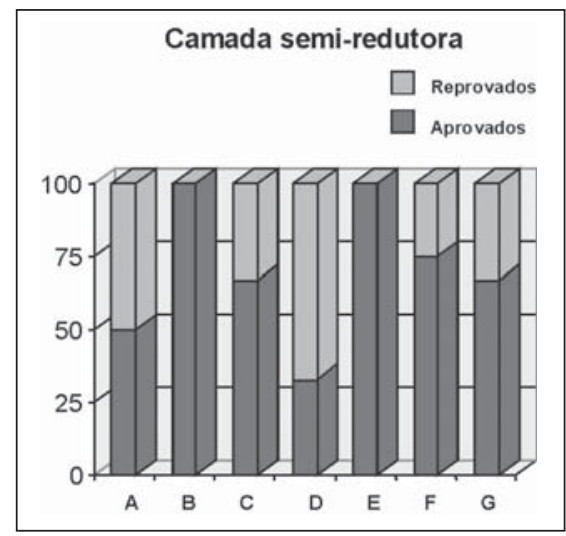

Gráfico 5. Porcentual de equipamentos aprovados e reprovados no teste de camada semi-redutora.

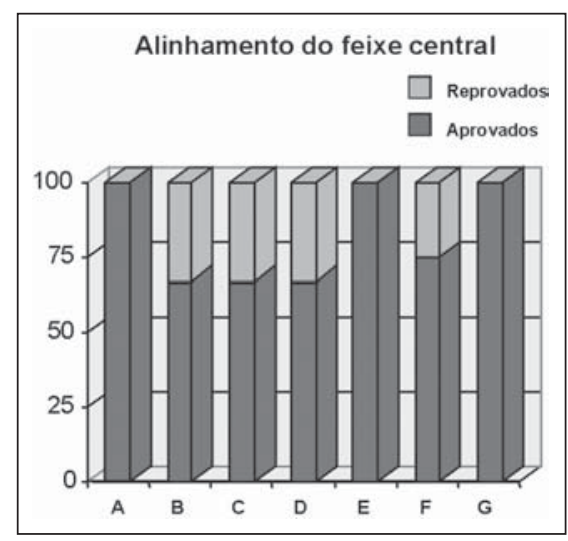

Gráfico 6. Porcentual de equipamentos aprovados e reprovados no teste de alinhamento do feixe central.

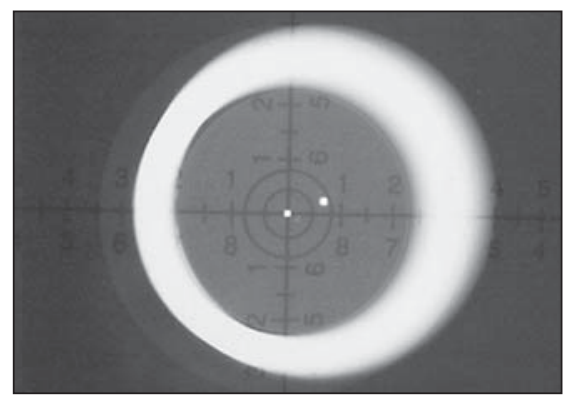

Figura 1. Imagem do feixe central desalinhado.

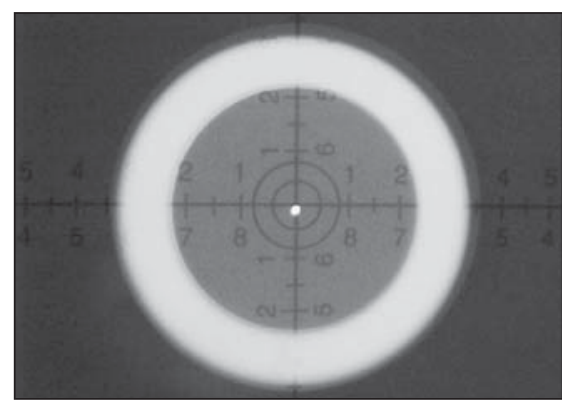

Figura 2. Imagem do feixe central alinhado. 


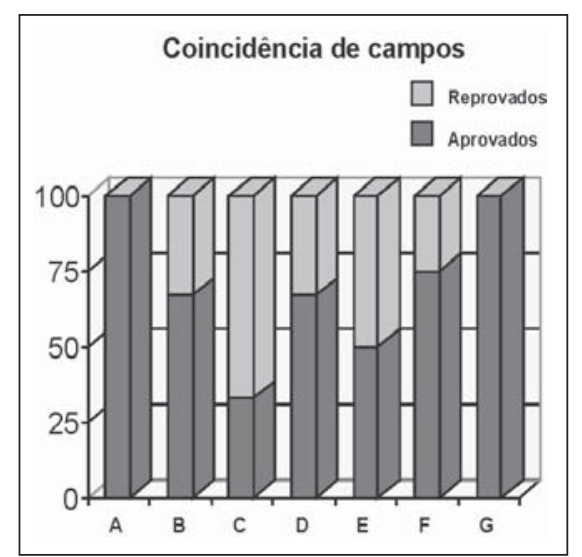

Gráfico 7. Porcentual de equipamentos aprovados e reprovados no teste de coincidência de campos.

Os resultados do teste de coincidência entre os campos de radiação e de luz podem ser vistos no Gráfico 7 .

Nas Figuras 3 e 4 temos dois exemplos do teste de coincidência de campos: o primeiro, de um equipamento que está inadequado com relação a este teste, e o outro, de um equipamento adequado.

Os resultados do teste de reprodutibilidade de exposição são mostrados no Gráfico 8 .

Com relação à linearidade de exposição, os resultados são apresentados no Gráfico 9.

$\mathrm{O}$ teste de rendimento do tubo de raios $\mathrm{X}$ foi o que obteve o pior desempenho de todos aqueles realizados neste trabalho. Apenas um de todos os equipamentos verificados apresentou valor de rendimento dentro dos parâmetros aceitáveis para este teste $^{(2,11,12)}$. O referido equipamento pertence ao SR F e deve ser destacado que ele havia sido instalado no SR, na ocasião dos testes, há menos de dois anos ${ }^{(\mathbf{1 3 )}}$.

Quanto ao teste de ponto focal, podemos verificar os resultados na Tabela 1.

Tabela 1 Porcentual de equipamentos aprovados e reprovados no teste de ponto focal.

\begin{tabular}{|c|c|c|}
\hline \multirow{2}{*}{ Hospitais } & \multicolumn{2}{|c|}{ Ponto focal } \\
\cline { 2 - 3 } & Aprovados & Reprovados \\
\hline A & $50 \%$ & $50 \%$ \\
B & $67 \%$ & $33 \%$ \\
C & $33 \%$ & $67 \%$ \\
D & $33 \%$ & $67 \%$ \\
E & $50 \%$ & $50 \%$ \\
F & $63 \%$ & $37 \%$ \\
G & $33 \%$ & $67 \%$ \\
\hline
\end{tabular}

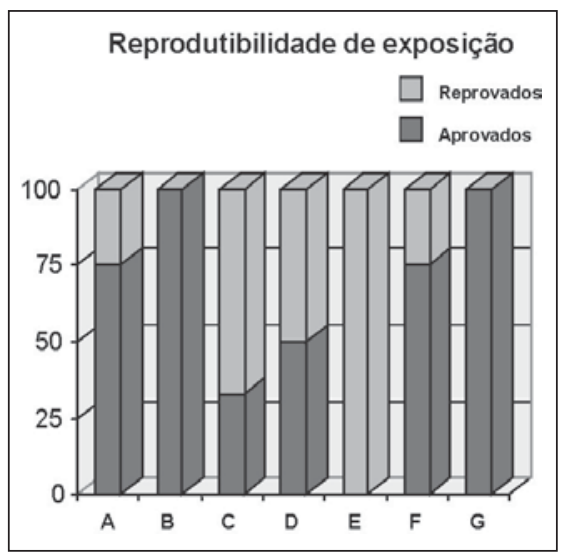

Gráfico 8. Porcentual de equipamentos aprovados e reprovados no teste de reprodutibilidade de exposição.

As Figuras 5 e 6 mostram imagens de pontos focais fora e dentro dos padrões aceitáveis.

Foi observado que $85 \%$ dos SR inspecionados apresentaram vestimentas de proteção individual em condições aceitáveis, e 50\% apresentaram número suficiente de vestimentas de proteção individual em uso. Os protetores de gônadas foram os mais au-

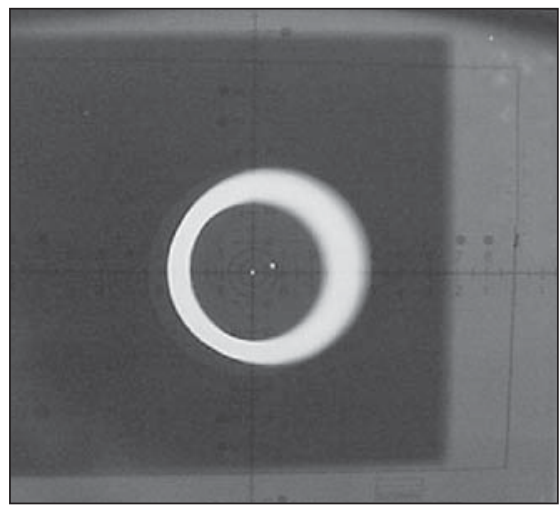

Figura 3. Imagem de coincidência de campos em desacordo.

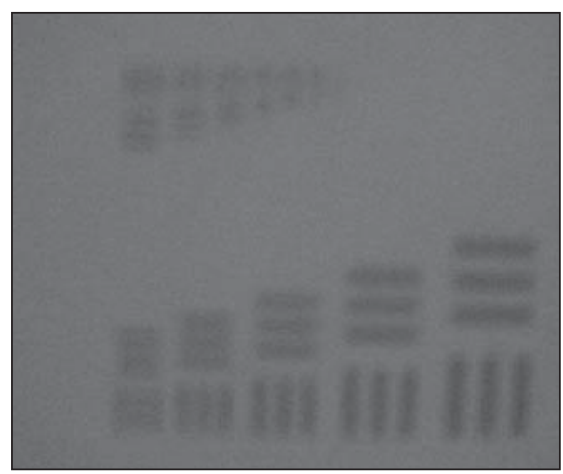

Figura 5. Imagem do ponto focal em desacordo.

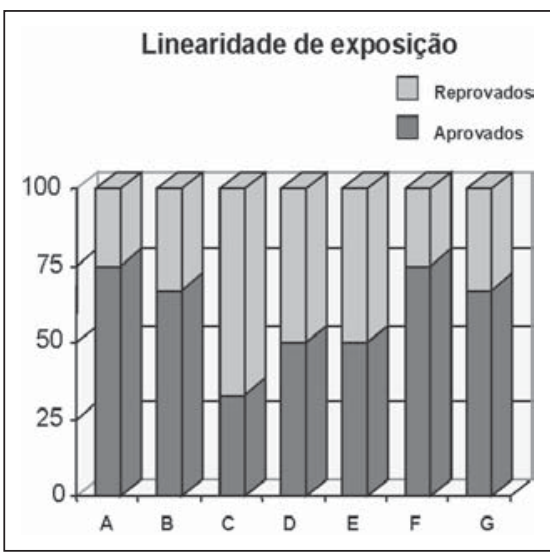

Gráfico 9. Porcentual de equipamentos aprovados e reprovados no teste de linearidade de exposição.

sentes das vestimentas de proteção individual. Foi verificado, também, que alguns aventais plumbíferos apresentavam rachaduras, devido ao mau acondicionamento.

Foram verificadas nove câmaras escuras neste trabalho, pois um dos SR tem três câmaras escuras. Neste caso foi medida a iluminância no interior das câmaras escuras de cada SR, tendo sido verificado que

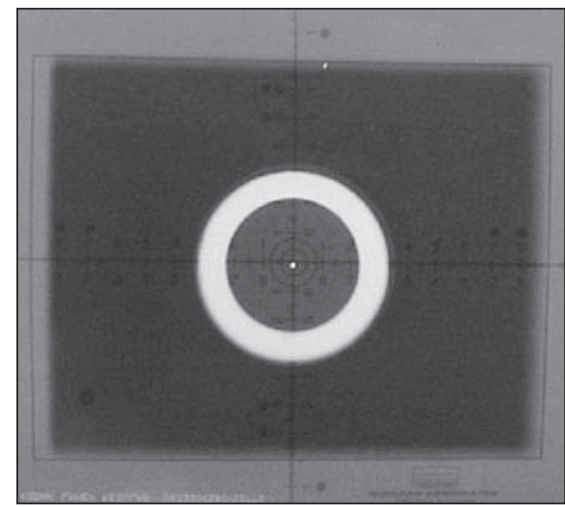

Figura 4. Imagem de coincidência de campos em acordo.

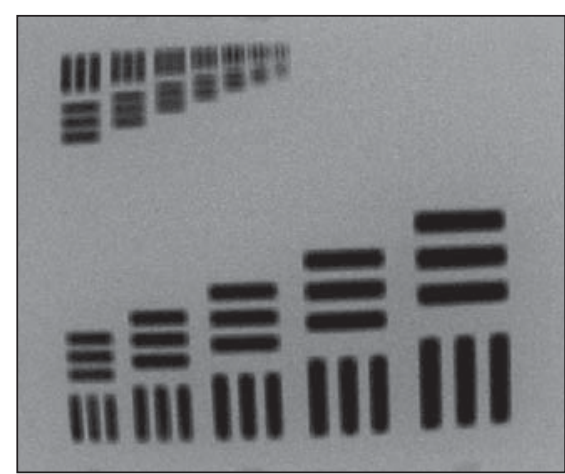

Figura 6. Imagem do ponto focal em acordo. 
todas se apresentaram dentro do recomendado $^{(\mathbf{1 4})}$. Apenas uma das câmaras escuras estava fora das especificações, sendo seu valor de iluminância aproximadamente cinco vezes o valor padrão. Este fato ocorreu devido a problemas na vedação da luz, propiciada por problemas estruturais no teto da câmara escura e pintura inadequada de sua entrada. Esta câmara foi colocada em reforma na ocasião.

Foram verificadas, também, a existência ou não de lanterna de segurança e as condições de higiene e limpeza das câmaras escuras. Foi observado que apenas 50\% das câmaras escuras tinham lanterna de segurança em condições adequadas e que estavam em efetivo uso.

Os resultados do teste de resolução de imagem propiciada pelo conjunto "écran"filme, realizado apenas em três SR, podem ser observados na Tabela 2 .

Tabela 2 Porcentual de "écrans"-filmes aprovados e reprovados no teste de resolução "écran"-filme.

\begin{tabular}{|c|c|c|}
\hline \multirow{2}{*}{ Hospitais } & \multicolumn{2}{|c|}{ Resolução "écran"-filme } \\
\cline { 2 - 3 } & Aprovados & Reprovados \\
\hline A & $75 \%$ & $25 \%$ \\
D & $80 \%$ & $20 \%$ \\
F & $70 \%$ & $30 \%$ \\
\hline
\end{tabular}

Foi verificado, no teste de alinhamento de grade, que todas as grades antiespalhadoras dos equipamentos de raios $\mathrm{X}$ dos $\mathrm{SR}$ avaliados são focadas, pois não houve diferença significativa entre as densidades ópticas obtidas e as esperadas. No entanto, foi verificado em um SR que um equipamento estava com a grade fora do campo focado de radiação, gerando uma imagem sem homogeneidade, o que foi corrigido posteriormente. As Figuras 7 e 8 mostram dois exemplos desse teste, um reprovado e outro aprovado. Na Figura 7 não existe simetria entre as densidades ópticas das imagens circulares todas em relação à circunferência central, enquanto na Figura 8 esta simetria é verificada.

Quanto ao teste de contato "écran"-filme, são apresentados dois exemplos: na Figura 9 observamos duas regiões, indicadas pelas setas, que apontam a falta de contato entre o "écran" e o filme, e na Figura 10 verificamos que a imagem é de um conjunto "écran"-filme em boas condições.

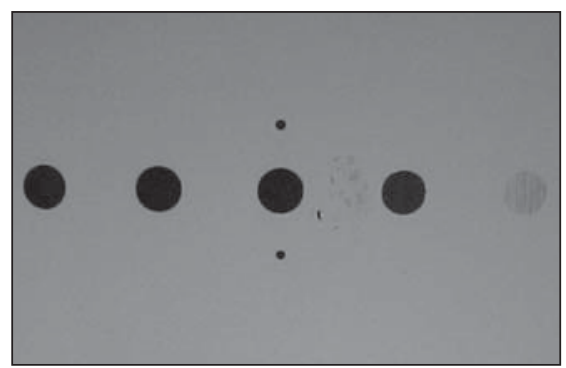

Figura 7. Imagem do teste de alinhamento de grade em desacordo.

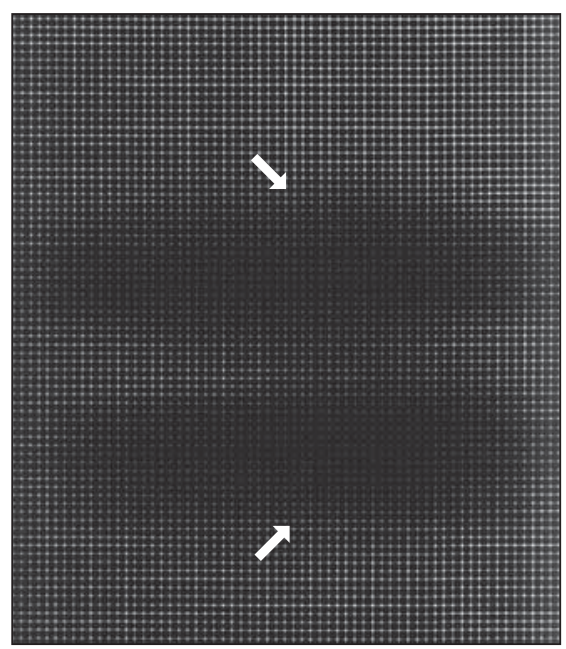

Figura 9. Imagem do teste de contato "écran"-filme em desacordo.

Dos chassis inspecionados, $95 \%$ estavam dentro das condições adequadas. Foi observado que um dos SR apresentou 5\% dos seus chassis com entrada de luz. A Figura 11 nos mostra um exemplo de um chassi com entrada de luz acentuada em uma das laterais. De todos os SR verificados, apenas um deles fazia limpeza sistemática, rotineira e com periodicidade programada dos "écrans" e chassis.

Os resultados dos testes realizados nos negatoscópios são mostrados na Tabela 3.

\section{DISCUSSÃO}

Nos testes de exatidão e reprodutibilidade de $\mathrm{kVp}$ obtivemos média de $55 \%$ dos equipamentos reprovados em exatidão e $15 \%$ reprovados em reprodutibilidade. $\mathrm{O}$ SR com o maior porcentual de equipamentos aprovados foi o B, com mais de $60 \%$ dos equipamentos aprovados em todos os parâmetros verificados neste teste. Os SR com pior desempenho foram o A e o D,

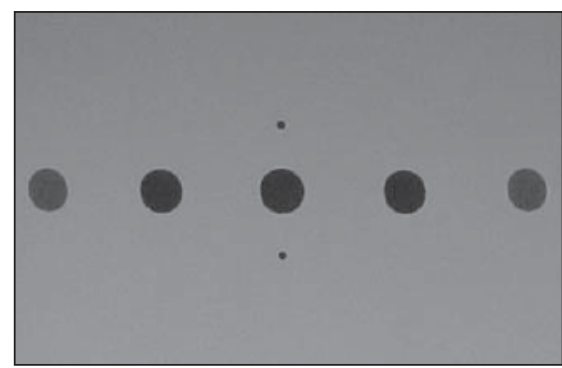

Figura 8. Imagem do teste de alinhamento de grade em acordo.

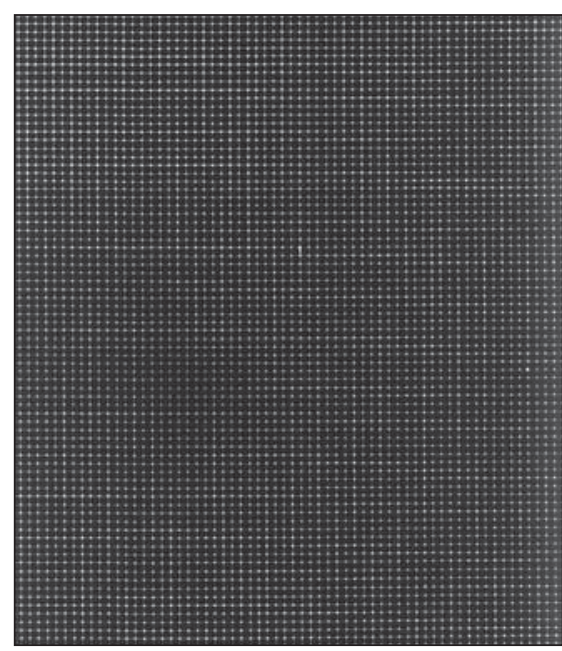

Figura 10. Imagem do teste de contato “écran"-filme em acordo.

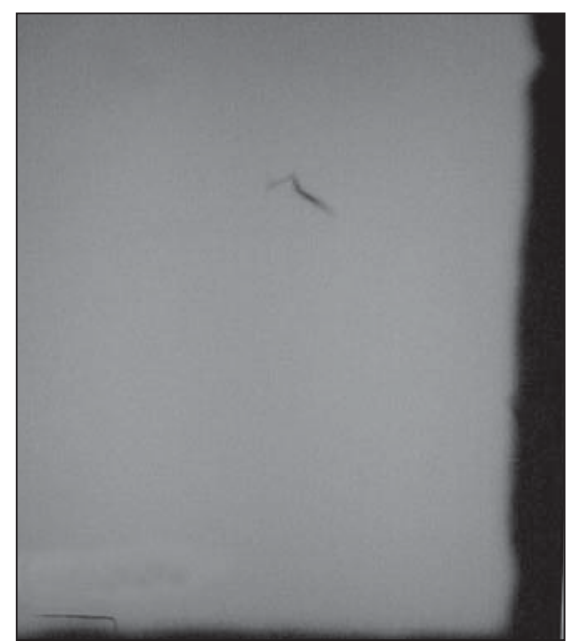

Figura 11. Exemplo de chassis com entrada de luz.

com pelo menos um dos parâmetros totalmente reprovado. Destacamos ainda o SR F, que apresentou desempenho acima dos $60 \%$, mas apenas para os valores de 60 e $100 \mathrm{kVp}$. Pela análise dos resultados podemos ver claramente que a faixa de $\mathrm{kVp}$ 
Tabela 3 Porcentual de negatoscópios aprovados e reprovados no teste de luminância.

\begin{tabular}{|c|c|c|}
\hline \multirow{2}{*}{ Hospitais } & \multicolumn{2}{|c|}{ Negatoscópios } \\
\cline { 2 - 3 } & Aprovados & Reprovados \\
\hline A & $15 \%$ & $85 \%$ \\
B & $20 \%$ & $80 \%$ \\
C & $10 \%$ & $90 \%$ \\
D & $25 \%$ & $75 \%$ \\
E & $45 \%$ & $55 \%$ \\
F & $30 \%$ & $70 \%$ \\
G & $15 \%$ & $85 \%$ \\
\hline
\end{tabular}

que apresentou pior desempenho no conjunto de SR verificados neste trabalho foi a faixa de $100 \mathrm{kVp}$. Segundo Gori et al. ${ }^{(\mathbf{1 5})}$, $42 \%$ dos equipamentos de raios $\mathrm{X}$ foram rejeitados nos testes de $\mathrm{kVp}$ devido à inexistência de um PGQ e, conseqüentemente, da execução de testes de CQ. Nas mesmas condições, Carrizales e Cozman ${ }^{(\mathbf{1 6})}$ verificaram que $61 \%$ dos equipamentos foram rejeitados, e Bacelar et al. ${ }^{(\mathbf{1 7 )}}$ encontraram $80 \%$. Com a implantação de um PGQ em que constavam testes de CQ, o porcentual agora encontrado por Bacelar et al. ${ }^{(\mathbf{1 7})}$ e por Medeiros e Alves ${ }^{(\mathbf{1 8})}$ foi de $0 \%$, enquanto Gori et al. ${ }^{(15)}$ encontraram $19 \%$ e Godechal et al. ${ }^{(\mathbf{1 2})}$ verificaram $15 \%$ em exatidão e $13 \%$ em reprodutibilidade.

Nos testes de exatidão e reprodutibilidade de tempo de exposição encontramos $53 \%$ dos equipamentos reprovados em exatidão e $31 \%$ reprovados em reprodutibilidade em todos os SR verificados. A partir da observação dos resultados é possível apontar que os tempos curtos apresentaram elevado porcentual de reprovação, tanto de exatidão quanto de reprodutibilidade de tempo de exposição, para cada uma das salas de cada SR. Com relação aos testes de tempo de exposição, Gori et al. ${ }^{(\mathbf{1 5})}$ apontam que $81 \%$ dos equipamentos de raios $\mathrm{X}$ foram rejeitados quando não havia um PGQ e, conseqüentemente, um CQ nos SR da Itália. Nas mesmas condições, Carrizales e Cozman ${ }^{(\mathbf{1 6})}$ verificaram que na Venezuela $75 \%$ dos equipamentos foram rejeitados, e Bacelar et al. ${ }^{(\mathbf{1 7 )}}$ encontraram $67 \%$ no Brasil. Com a implantação de um PGQ em que constavam testes de $\mathrm{CQ}$, o porcentual agora encontrado por Bacelar et al. ${ }^{(\mathbf{1 7 )}}$ foi de $0 \%$, enquanto Gori et al. ${ }^{(15)}$ encontraram $22 \%$, Godechal et $a l .{ }^{(12)}$ verificaram $20 \%$, e Medeiros e Al- ves $^{(18)}, 24 \%$. Pudemos constatar que, com relação às medidas realizadas neste trabalho, não foram observadas melhorias no desempenho dos equipamentos quanto ao tempo de exposição de um dos SR que havia sido testado algum tempo antes ${ }^{(\mathbf{1 9 , 2 0})}$.

Para os testes de kVp e tempo de exposição foram realizadas medidas em diferentes valores de mA para cada um dos valores de tempo de exposição, quando o equipamento nos permitia, pois foram observados desvios de até $10 \%$ em relação às medidas feitas quando não se variava esse parâmetro. Este procedimento se mostrou necessário, uma vez que torna mais confiáveis as medidas de $\mathrm{kVp}$, sendo adotado e incorporado em todos os testes de tempo de exposição.

Com relação à CSR, observamos que $30 \%$ dos SR verificados estão fora dos parâmetros aceitáveis ${ }^{(3,11)}$. Este porcentual leva em consideração um valor mínimo de filtração $^{(3,11)}$, no entanto destacamos que uma filtração muito acima do recomendado pode acarretar problemas, tais como a diminuição do rendimento do tubo de raios $\mathrm{X}^{\mathbf{( 9 , 1 1 )}}$. Carrizales e Cozman ${ }^{(\mathbf{1 6})}$ verificaram que $46 \%$ dos equipamentos sem um PGQ foram reprovados no teste de CSR. Gori et al. ${ }^{(\mathbf{1 5})}$ verificaram que o porcentual de equipamentos reprovados neste teste era de $11 \%$ antes da implantação de um PGQ e que caiu para 6\%, o mesmo valor encontrado por Godechal et al. ${ }^{(\mathbf{1 2})}$.

No teste de alinhamento do feixe central, 17,7\% dos equipamentos foram reprovados. Um desvio acentuado no feixe central de raios $\mathrm{X}$ acarreta um possível erro ao se radiografar com precisão uma determinada área. De acordo com Gori et al. ${ }^{(15)}$, o porcentual de equipamentos rejeitados neste teste é de 3\% quando existe um PGQ implantado no SR, contra 35\% sem qualquer CQ. Já Bacelar et al. ${ }^{(\mathbf{1 7})}$ dizem que este valor é de $5 \%$ contra $85 \%$ sem CQ, enquanto Godechal et al. ${ }^{(\mathbf{1 2})}$ encontraram 0\% nas mesmas condições. Carrizales e Cozman $^{(\mathbf{1 6})}$ observaram um porcentual de $53 \%$ de equipamentos rejeitados quando não se dispunha de um PGQ.

No teste de coincidência de campos, $30 \%$ dos equipamentos foram reprovados. Um erro acentuado neste parâmetro acarreta um possível erro ao se radiografar com precisão os limites de uma determinada área $^{(\mathbf{2 1})}$. De acordo com Gori et al. ${ }^{(\mathbf{1 5})}$, o porcentual de equipamentos rejeitados neste teste é de $13 \%$; já Bacelar et al. ${ }^{(17)}$ dizem que este valor é de $5 \%$, enquanto Godechal et al. ${ }^{(\mathbf{1 2})}$ encontraram $0 \%$, sendo que em todas as situações existia um PGQ implantado no SR. Carrizales e Cozman ${ }^{(\mathbf{1 6})}$ observaram porcentual de $41 \%$ de equipamentos rejeitados neste teste, e Medeiros e Alves ${ }^{(\mathbf{1 8 )}}$ encontraram $17 \%$, ambos quando não se dispunha de um PGQ.

De acordo com os resultados do teste de reprodutibilidade de exposição, 38\% dos equipamentos verificados foram rejeitados. Carrizales e Cozman ${ }^{(\mathbf{1 6})}$ verificaram que $33 \%$ dos equipamentos foram reprovados no teste de reprodutibilidade de exposição em SR que não tinham um PGQ, enquanto Godechal et al. ${ }^{(\mathbf{1 2})}$ observaram que quando se tem um PGQ implantado no SR este valor é de $1,3 \%$.

No teste de linearidade de exposição verificamos que $40 \%$ dos equipamentos foram reprovados. Antes de implantar um PGQ, Bacelar et al. ${ }^{(17)}$ verificaram que $66 \%$ dos equipamentos foram reprovados no teste de linearidade de exposição; após terem implantado o PGQ, todos os equipamentos foram aprovados neste teste. Nessa mesma condição de um SR com um PGQ, Godechal et al. $^{(\mathbf{1 2})}$ verificaram apenas 3\% de equipamentos reprovados.

A análise do rendimento é um dos principais indicadores para que possamos verificar se o desempenho real do equipamento está próximo daquele que foi projetado. Para os equipamentos de raios $\mathrm{X}$, os parâmetros que influenciam na determinação do rendimento são: $\mathrm{kVp}$, tempo de exposição, mA (linearidade e reprodutibilidade) e a CSR. Gori et al. ${ }^{(15)}$ e Godechal et al. ${ }^{(\mathbf{1 2})}$ verificaram que, em equipamentos com idade acima de dez anos, o desempenho dos parâmetros físicos, principalmente o rendimento, é bem inferior, exigindo que se faça um controle maior desses equipamentos para que eles possam ser utilizados devidamente.

Com relação ao teste de ponto focal, podemos verificar rejeição de $64 \%$ dos equipamentos. Este fato compromete diretamente a qualidade das imagens obtidas, uma vez que não se consegue observar nas radiografias detalhes menores que a capacidade de resolução do equipamento ${ }^{(\mathbf{7 , 9 , 1 2})}$. 
Outro fator que contribui para esse número elevado de equipamentos reprovados é a idade dos equipamentos ${ }^{(12,15)}$.

Foram observados, em cada um dos SR, a existência, as condições de uso e o armazenamento, e na medida do possível, a freqüência com que eram utilizadas as vestimentas de proteção individual em cada um dos SR que foram objeto deste trabalho. No entanto, pudemos verificar que, de modo geral, ainda não existe uma cultura de proteção radiológica nos profissionais que se utilizam de radiação ionizante em exames de raios $\mathrm{X}$ diagnóstico. Foi uma constante observarmos que aventais plumbíferos são guardados de maneira inadequada, podendo provocar rachaduras em sua estrutura, não impedindo assim a passagem dos raios X. Foi observado, também, que, com relação à grande maioria dos acompanhantes que precisam conter os pacientes na hora de um exame de raios $\mathrm{X}$, não lhes é recomendado o uso de vestimenta de proteção individual, o que acarreta exposição desnecessária desse segmento da população.

As câmaras escuras dos SR foram aprovadas, com índices de iluminância aceitáveis, o que influencia diretamente na qualidade da imagem ${ }^{(\mathbf{1 4})}$. No entanto, apenas um dos SR avaliados cumpria as especificações nacionais quanto à colocação adequada de lanternas de segurança. A grande maioria dos SR verificados continha uma grande quantidade de objetos desnecessários ou obsoletos dentro das câmaras escuras (rádio, quadros, vassoura, material de limpeza, embalagens vazias de produtos químicos, cabides com roupas, colchonetes, mochilas, etc.), o que contribui para o aumento de poeira no interior das câmaras, e que certamente poderá se depositar sobre filmes e "écrans", interferindo na qualidade da imagem. Dos SR verificados, apenas o que pertence ao hospital E apresentou um plano de limpeza periódica em suas câmaras escuras.

No teste de resolução "écran"-filme, $25 \%$ dos "écrans" foram reprovados. Este fato certamente se deu devido à vida útil desses "écrans".

Dos SR verificados, apenas um apresentou problemas no teste de alinhamento de grade. Neste SR, um dos equipamentos apresentou desvio da grade antiespalha- dora. Este fato pode interferir no exame, de forma a ocasionar falta de homogeneidade na imagem.

Quanto à falta de integridade de chassis e "écrans", podemos apontar como duas das principais causas a sua má utilização e o excessivo tempo em que eles ficam em uso nos SR.

Em grande parte dos SR verificados, por se tratar de hospitais públicos de grande porte, foi observado que, de rotina, são realizados muitos exames seguidos, sem que sejam revelados os filmes, e só após algum tempo é que estes são levados à câmara escura para revelação. Este fato é justificado por parte dos SR como sendo para agilizar o trabalho. No entanto, estes chassis são levados, muitas vezes em carrinhos similares àqueles usados em supermercados, onde são, na maioria das vezes, mal acondicionados. Este procedimento acarreta diminuição da vida útil dos chassis, ocasionando entrada de luz, emperramento das travas de fechamento, rachaduras, empenamento, etc. Este procedimento, sendo repetido por um longo tempo, certamente compromete a integridade desses equipamentos

Quantos aos "écrans", foi verificado, com o auxílio de lâmpada ultravioleta, que eles apresentaram muita sujeira depositada em sua superfície. Como esta sujeira é imperceptível à luz branca, conseqüentemente não é removida. Faz-se necessário treinamento adequado aos profissionais que limpam os "écrans" para que consigam, com lâmpada adequada, visualizar a sujeira depositada sobre o “écran”. Dos SR verificados, todos apresentaram sujeira ao serem observados adequadamente. Isto ocorreu porque os "écrans" pareciam estar limpos, o que aparentemente não justificava uma limpeza mais efetiva.

Os negatoscópios avaliados neste trabalho apresentaram média de luminância abaixo dos padrões estabelecidos interna-

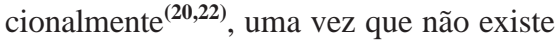
padronização nacional para a sua construção. No entanto, este fato é agravado pela falta de limpeza periódica, demora na reposição de lâmpadas queimadas, utilização de lâmpadas fluorescentes com diferentes cores do espectro da luz branca (luz do dia, amarela, azul, etc.). Foram observados negatoscópios com lâmpadas frias de diferen- tes espectros de cores, o que faz com que a superfície do negatoscópio fique sem homogeneidade luminosa. Foram observados, também, negatoscópios com a superfície de acrílico rachada, suja e manchada, assim como lâmpadas aparentando a idade útil expirada. Também não foi observado nenhum procedimento sistemático de limpeza e manutenção desses equipamentos.

As câmaras claras apresentaram, em sua maioria, elevado índice de iluminância, o que prejudica o trabalho do radiologista, especialmente a elaboração do laudo radiográfico $^{(\mathbf{1 4})}$, porque gera maior cansaço e fotofobia.

\section{CONCLUSÕES}

A partir da aplicação e execução dos testes de CQ, foi possível verificar que as condições dos SR que foram avaliados neste trabalho se encontram, de modo geral, abaixo dos limites mínimos requeridos pelos protocolos nacionais e internacionais de CQ, tanto no aspecto quantitativo, na realização de medidas nos parâmetros físicos dos equipamentos e acessórios, quanto no aspecto qualitativo, de se inserir na rotina de trabalho procedimentos que visem a uma melhoria progressiva da qualidade da imagem, e dos parâmetros de qualidade mínimos exigidos pelos dispositivos legais do nosso país. Este fato se dá tanto por desconhecimento específico dos profissionais envolvidos quanto por não se ter um PGQ que garanta a implementação de rotinas de qualidade que visem à melhoria das imagens diagnósticas, acompanhada da redução de custos do SR, assim como, e não menos importante, reduzir a dose nos pacientes e nos profissionais que são ocupacionalmente expostos.

A medida do desempenho dos SR, segundo os protocolos de testes utilizados, foi suficiente para indicar que o cumprimento dos dispositivos legais já aponta para uma melhoria progressiva e sistemática da qualidade dos SR, e que o cumprimento do dispositivo legal deve se dar não apenas por imposição legal, mas também como requisito mínimo de se garantir bom desempenho do SR.

Os resultados obtidos neste trabalho são fruto direto da implementação de testes de CQ, e que estes devem compor um 
PGQ mais amplo e abrangente. É de inteira pertinência que seja sugerido neste trabalho a implementação desses testes por um profissional qualificado, para que sejam minimizados os graus de incerteza das diversas etapas da obtenção das imagens radiológicas. Pode ser compreendido também que a relação custo-benefício torna relevante a implantação e manutenção dos procedimentos contidos e executados neste trabalho.

\section{Agradecimentos}

Os autores agradecem a Capes, Faperj e HUCFF-UFRJ, pelo apoio financeiro destinado à realização deste trabalho.

\section{REFERÊNCIAS}

1. Rehani MM. Diagnostic imaging quality assurance New Delhi, India: Jaypee Brothers Medical Publishers, 1994.

2. Moores BM, Watkinson AS, Henshaw ET, Pearcy BJ. Quality control in diagnostic radiology. In Oberhofer $\mathrm{M}$, ed. Advances in radiation protection. 1st ed. Brussels and Luxembourg: Kluwer Academic Publishers, 1991:209-36.

3. Brasil. Ministério da Saúde. Secretaria de Vigilância Sanitária. Diretrizes de proteção radiológica em radiodiagnóstico médico e odontológico. Portaria no 453. Brasília, DF: Diário Oficial da União, 2/6/ 1998 .

4. Linton OW, Properzio WS, Steele JP. Quality assur- ance: an idea whose time has come. AJR 1979;133: 989-92.

5. Périard MA, Chaloner P. Diagnostic X-ray imaging quality assurance: an overview. Can J Med Radiat Technol 1996;27:171-7.

6. Yacovenco A, Lira SH, Borges JC, Mota HC. Programa de garantia de qualidade em radiologia diagnóstica. RBE/CEB 1994;10:7-19.

7. Craveiro PM, Nishimura R, Nascimento EFR, Stefano EJ, Santos JB. Elaboração de um protocolo de controle de qualidade em radiodiagnóstico. Rev Imagem 1999;21:161-8.

8. International Commission on Radiological Protection. Recommendations of the International Commission on Radiological Protection. Oxford: Pergamon Press. ICRP Publication n. 26, Ann ICRP 1977;1(3).

9. Protocolo de avaliação de proteção radiológica em radiodiagnóstico médico. Rio de Janeiro, RJ: RXDURMCF, IRD/CNEN, 2000.

10. Moeller D. History and perspective on the development of radiation protection standards. In: Sinclair WK, ed. Radiation protect today: the NCRP at sixty years-proceedings of the twenty-fifth annual meeting. Bethesda, MD: National Council on Radiation Protection and Measurements, 1990.

11. Papp J. Quality management in the imaging sciences. St. Louis, MO: Mosby, 1998

12. Godechal D, Delhove J, Mambour C, Coomans J, Wambersie A. A quality assurance programme for medical X ray diagnostic units carried out in Belgium. Radiat Prot Dosimetry 1995;57:309-13.

13. Silva MO, Carvalho ACP, Oliveira SR, Magalhães LAG, Azevedo, ACP. Testes de aceitação em equipamento de raios X realizado no Hospital Universitário Clementino Fraga Filho. Anais Jornada Carioca de Radiologia, 2001.
14. Magalhães LAG. Controle de qualidade de processadoras automáticas. (Dissertação de Mestrado). Rio de Janeiro, RJ: Universidade Federal do Rio de Janeiro, 2001.

15. Gori C, Belli G, Calvagno S, et al. Quality control in the radiological departments of the Florence general hospital. Radiat Prot Dosimetry 1995;57: 315-6.

16. Carrizales LI, Cozman A. Quality control in radiodiagnosis in Venezuela, 1989-1990. Phys Med 1990;6:255-7.

17. Bacelar A, Oliveira SS, Streck EE, Borges V, Furtado APA, Pinto ALA. Avaliação preliminar de parâmetros operacionais em equipamentos fixos de raios X diagnóstico. Radiol Bras 1998;31:129-33.

18. Medeiros RB, Alves FFR. Análise dos resultados do programa de gerenciamento da qualidade dos equipamentos radiológicos. Rev Imagem 1997; 19: 97-9.

19. Almeida M, Guedes G, Magalhães LAG. Levantamento do atendimento no serviço de radiologia do HUCFF. X Jornada de Iniciação Científica da UFRJ, Rio de Janeiro, novembro de 1998.

20. Azevedo ACP, Yacovenco A, Gonçalves OD, Koch HA, Tonomura ET. Avaliação do funcionamento do serviço de radiodiagnóstico do Hospital Universitário Clementino Fraga Filho - Universidade Federal do Rio de Janeiro. Radiol Bras 1999;32:309_ 14.

21. Klimpel H, Kreienfeld H, Overbeck R. Field size and centring for conventional $\mathrm{X}$-ray equipment. BIR Report 18, 1989.

22. Azevedo ACP, Gonçalves OD, Magalhães LAG, Silva MO, Oliveira SR. Quality control of viewing boxes at the University Hospital of the Universidade Federal do Rio de Janeiro. Physica Medica 2001; XVII:89-92. 